\title{
Diagnosis and Individualized Treatment of Secondary Central Nervous System Lymphoma: A Case Report
}

\author{
Qian $\mathrm{Li}^{1}$ \\ Wei $\mathrm{Liu}^{2}$ \\ $\mathrm{Kai} \mathrm{Li}^{3}$ \\ Yifu $\operatorname{Tian}^{4}$ \\ Huan $\mathrm{Li}^{2}$ \\ 'Lung Hospital, Changsha Central \\ Hospital, University of South China, \\ Changsha, Hunan Province, People's \\ Republic of China; ${ }^{2}$ Department of \\ Oncology, Xiangya Hospital, Central \\ South University, Changsha, Hunan \\ Province, People's Republic of China; \\ ${ }^{3}$ Department of Radiology, Xiangya \\ Hospital, Central South University, \\ Changsha, Hunan Province, People's \\ Republic of China; ${ }^{4}$ Department of \\ Pathology, Xiangya Hospital, Central \\ South University, Changsha, Hunan \\ Province, People's Republic of China
}

Correspondence: Huan Li Department of Oncology, Xiangya Hospital, Central South University, Changsha, Hunan Province, People's Republic of China

Tel +8615II6337538

Email csulihuan@csu.edu.cn

\begin{abstract}
Non-Hodgkin lymphoma can disseminate to the central nervous system at initiation of treatment for systemic lymphoma or spread during the relapse of systematic lymphoma with CNS involvement, which is defined as secondary central nervous system lymphoma (SCNSL). The incidence of SCNSL depends on the pathological type of lymphoma and is especially high in aggressive lymphoma. SCNSL has a poor prognosis because of the lack of effective treatment regimens. This article presents a rare case of SCNSL; an individualized treatment regimen was designed according to the genetic analyses of the patient tumor and included a Bruton's tyrosine kinase (BTK) inhibitor. After six cycles of treatment and another two cycles of rituximab, most lesions lost their metabolic activity. However, in the final stage of treatment, our patient unfortunately suffered from respiratory failure, which revealed that we should pay attention to Pneumocystis jirovecii pneumonia during ibrutinib treatment.
\end{abstract}

Keywords: secondary central nervous system lymphoma, diffuse large B cell lymphoma, ibrutinib, individualized therapy, Pneumocystis jirovecii pneumonia

\section{Introduction}

The incidence of secondary nervous system lymphoma (SCNSL) is low, and most patients experience relapse. According to previous articles, only $2-10 \%$ of nonHodgkin's lymphoma patients were reported to have CNS involvement. ${ }^{1-4}$ Pathologic stratification revealed that high-grade lymphoma has a higher risk of CNS involvement than low-grade lymphoma, and the risk for Burkitt lymphoma and lymphoblastic lymphoma is especially high. The risk factors also include advanced disease, high levels of serum lactate dehydrogenase (LDH) and the International Prognostic Index. Schmitz et al have established and validated a risk score for it called Central Nervous System International Prognostic Index (CNSIPI) since $2016 .{ }^{5}$ The risk model has been widely used now for predicting central nervous system relapse in patients with diffuse large B-cell lymphoma treated with R-CHOP.

Because of the rarity of this disease, the best treatment regimen for SCNSL is still unknown, and most reports are based on retrospective studies. This paper summarizes previous treatment trials and advocates for the use of new combination drugs that provide patients with individualized therapy with good clinical outcomes. This patient agreed to let us collect her personal information for research and publication. 


\section{Case Presentation}

A 53-year-old married female with a medical history of diabetes was sent to our hospital because of daily persistent headaches in August 2018. Without any positive physical neurological examinations, a contrast-enhanced magnetic resonance imaging (MRI) scan of the brain suggested multiple enhancing masses in the corpus callosum and around the ventricles (Figure 1A-C). To make an accurate diagnosis, an
A

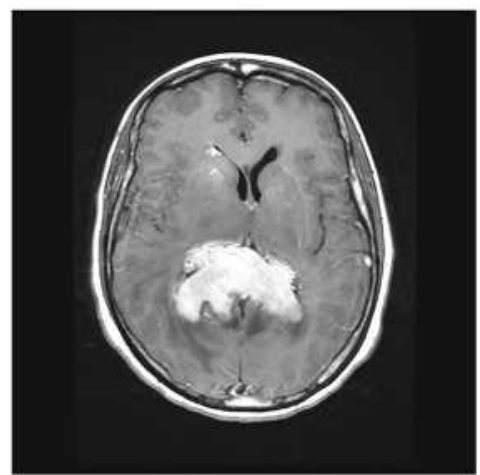

D

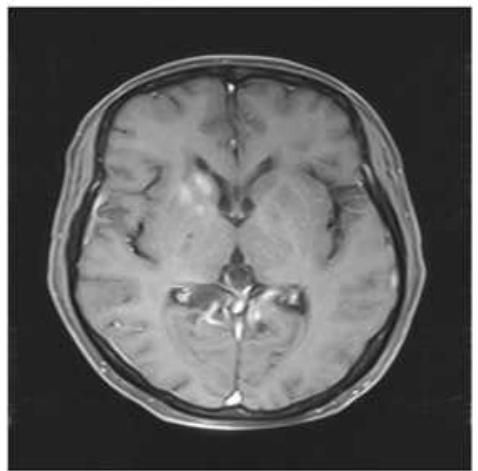

G

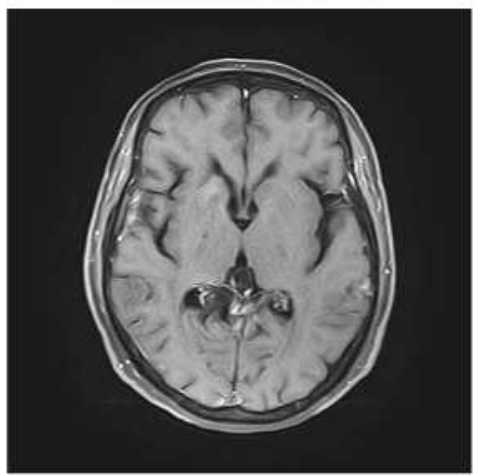

$\mathbf{J}$

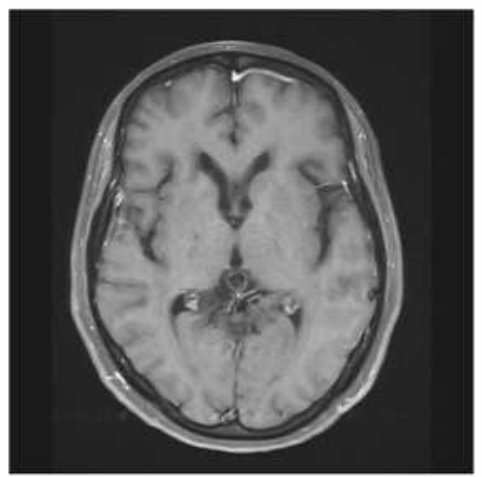

B

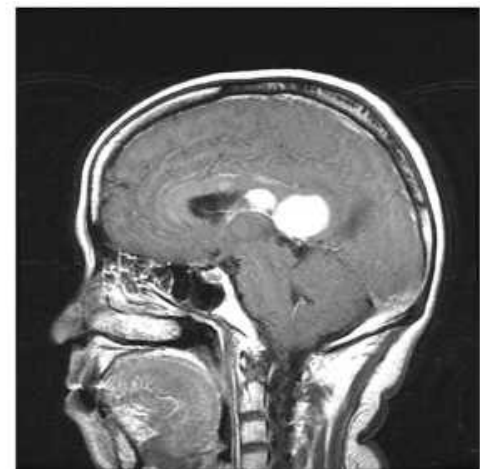

E

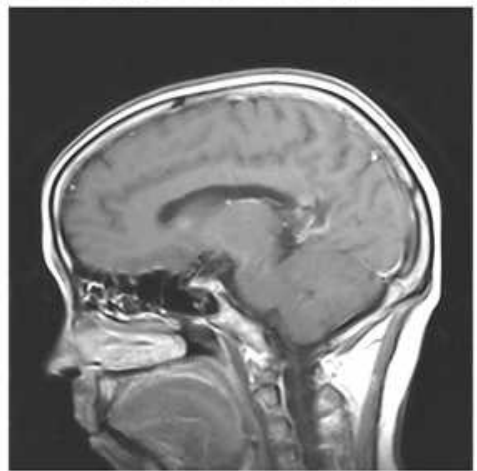

H

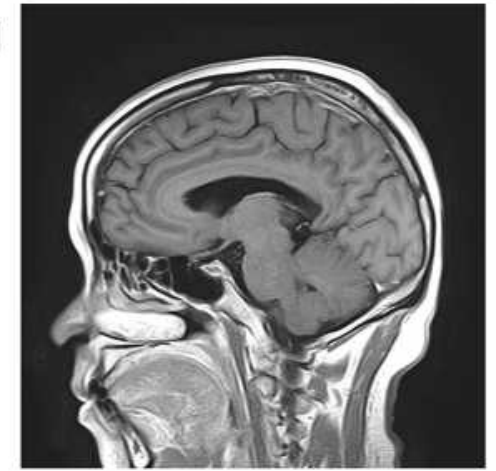

K

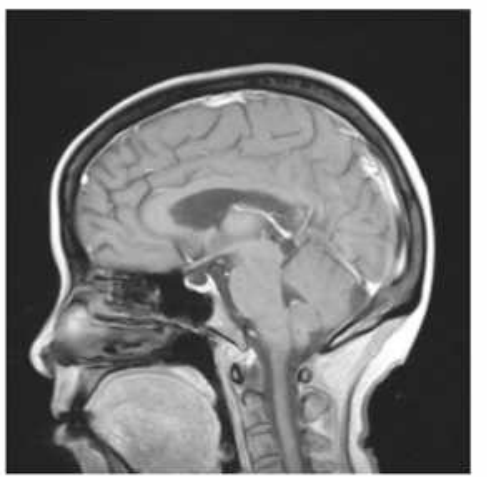

C

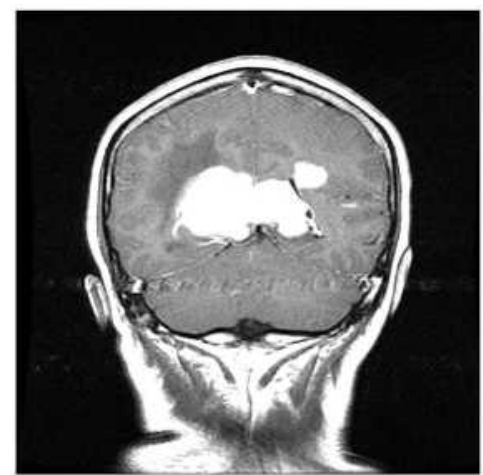

$\mathbf{F}$

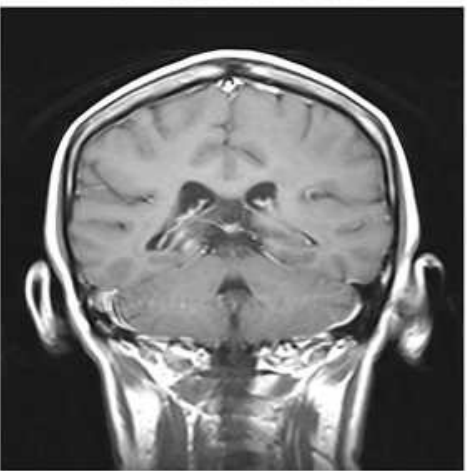

I

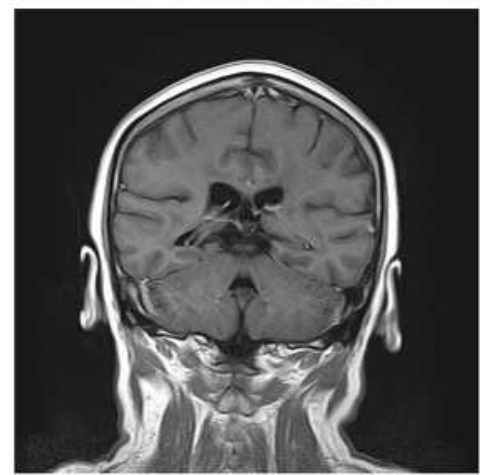

L

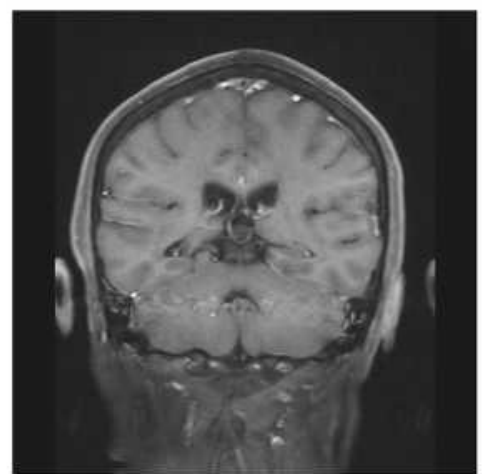

Figure I Different treatment periods of cranial MRI. (A-C) Multiple enhancing masses were shown in the corpus callosum and around the ventricles before our interventions. (D-F) Partial response after two cycles of treatment. (G-I) No obvious masses after finishing our treatment. (J-L) 30 months follow-up. 

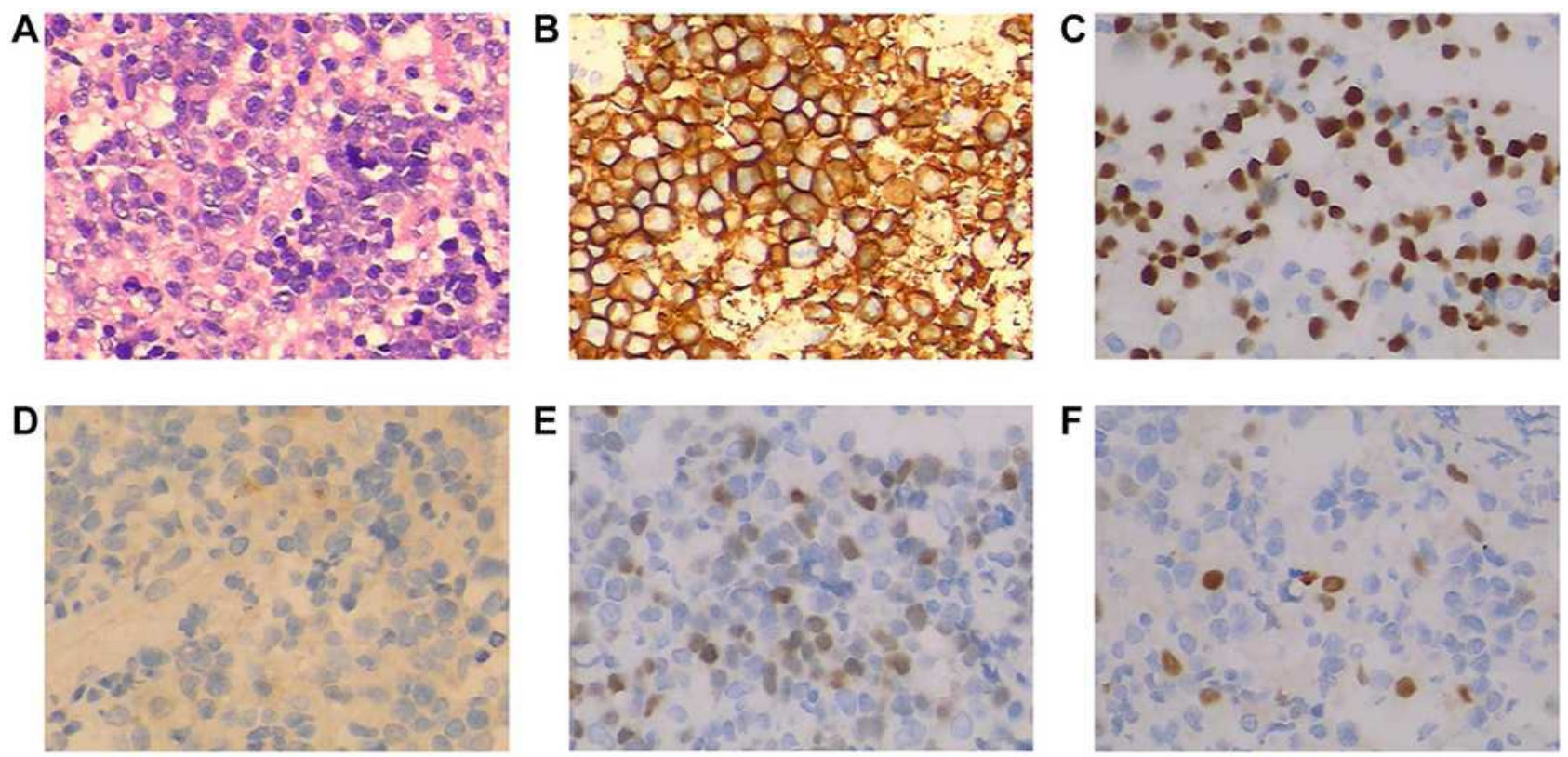

Figure 2 (A) Haematoxylin-eosin (H\&E) staining of biopsy samples (40x magnification). Immunohistochemical staining showed that tumor cells were positive for CD20 (B), PAX-5 (C) and MUMI (E), but not for CD30 (D) and Cyclin DI (F).

imaging-guided stereotactic needle biopsy was performed. Pathology (Figure 2) confirmed diffuse large B cell lymphoma (DLBCL; non-germinal center B cell (GCB)) after observing infiltrating neoplastic lymphoid cells that expressed CD45RO, CD20, PAX-5, MUM1, and Bcl-6 and that did not express CD3, CD10, CD30, cyclin D1, EBER (in situ hybridization), CD5, GFAP, CK-Pan and TdT (Ki-67 + approx. $70 \%$ of cells). 18F-fluorodeoxyglucose (FDG) positron emission tomography/computed tomography (PET/CT) was carried out, and the results demonstrated that many peripheral lymph nodes and extranodal organs or sites other than her brain, such as axillary lymph nodes, inferior diaphragmatic lymph nodes, and lymph nodes around the kidney area, were involved. Moreover, the vertebral arch of L3, sacrum, left side of the acetabulum, left femoral head and right femoral neck also showed FDG uptake. Our patient had negative cerebral spinal fluid (CSF) test results and negative marrow tests based on morphology and flow cytometry. Her LDH level was above the upper limit of normal, and an HIV test was negative. The genetic features of biopsy samples suggested MGMT (O6methylguanine-DNA methyltransferase) promoter methylation and $C D 79 B$ and $M y D 88 L 265 P$ mutations.

Considering the central nervous system (CNS) involvement of systemic DLBCL at the initial diagnosis and MGMT promoter methylation, we designed a methotrexate (MTX)-based immunochemotherapy regimen by referring to the MARIETTA trial (NCT02329080) ${ }^{6}$ which was sponsored by International Extranodal Lymphoma Study Group (IELSG) and we decided to combine two standardized chemotherapy regimens, high dose methotrexate-based regimen (MT-R: methotrexate, temozolomide and rituximab) ${ }^{7}$ for intracranial lesions and traditional R-CHOP (rituximab, vincristine, cyclophosphamide, prednisone and anthracycline $)^{8}$ immunochemotherapy regimen for extracranial lesions. Eventually, the details of the first cycle treatment for our patient were as follows: rituximab $\left(375 \mathrm{mg} / \mathrm{m}^{2}\right.$, day (d) 0$)$; high-dose intravenous MTX $\left(3.5 \mathrm{~g} / \mathrm{m}^{2}, \mathrm{~d} 1\right)$ with leucovorin for rescue, as previously described; ${ }^{9}$ vincristine $(2 \mathrm{mg}, \mathrm{d} 3)$; cyclophosphamide $\left(0.75 \mathrm{~g} / \mathrm{m}^{2}\right.$ once per day, $\left.\mathrm{d} 3-4\right)$; prednisone $(60 \mathrm{mg}, \mathrm{d} 3-7)$; and temozolomide $\left(150 \mathrm{mg} / \mathrm{m}^{2}\right.$ once per day, d3-7) for the first chemotherapy cycle (Figure 3, chemotherapy regimen A). Because anthracyclines cannot penetrate the blood-brain barrier and produce severe bone marrow suppression, which may affect the intracranial chemotherapy dose, we omitted the anthracyclines that were also adopted in the SCNSL1 trial in these treatment regimens. ${ }^{10}$ Essential supportive treatments were added, which included hydration and the alkalization of the urine before/after MTX infusion, ranitidine hydrochloride and granulocyte colony-stimulating factor (G-CSF). In the second cycle of our patient's treatment, considering 


\section{$\bigcirc$ methotrexate $\square$ ibrutinib $\square$ temozolomide}

chemotherapy regimen A

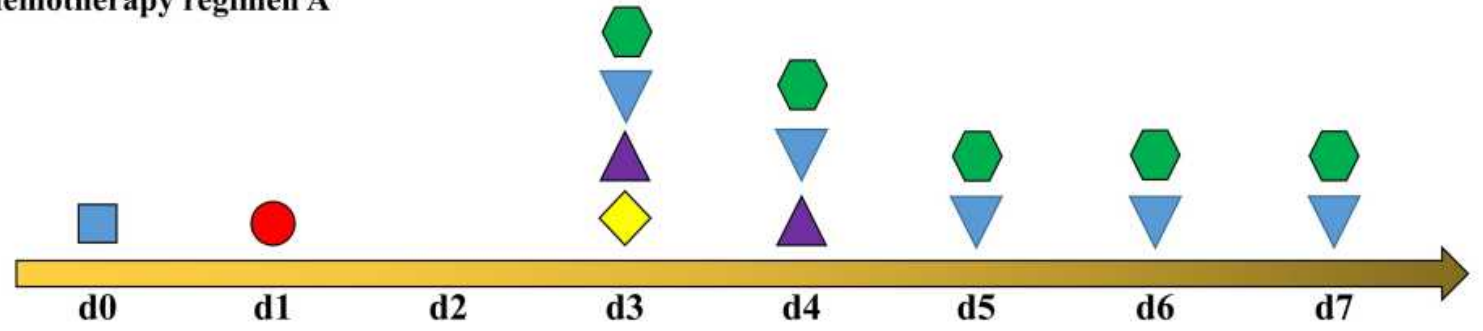

chemotherapy regimen $B$

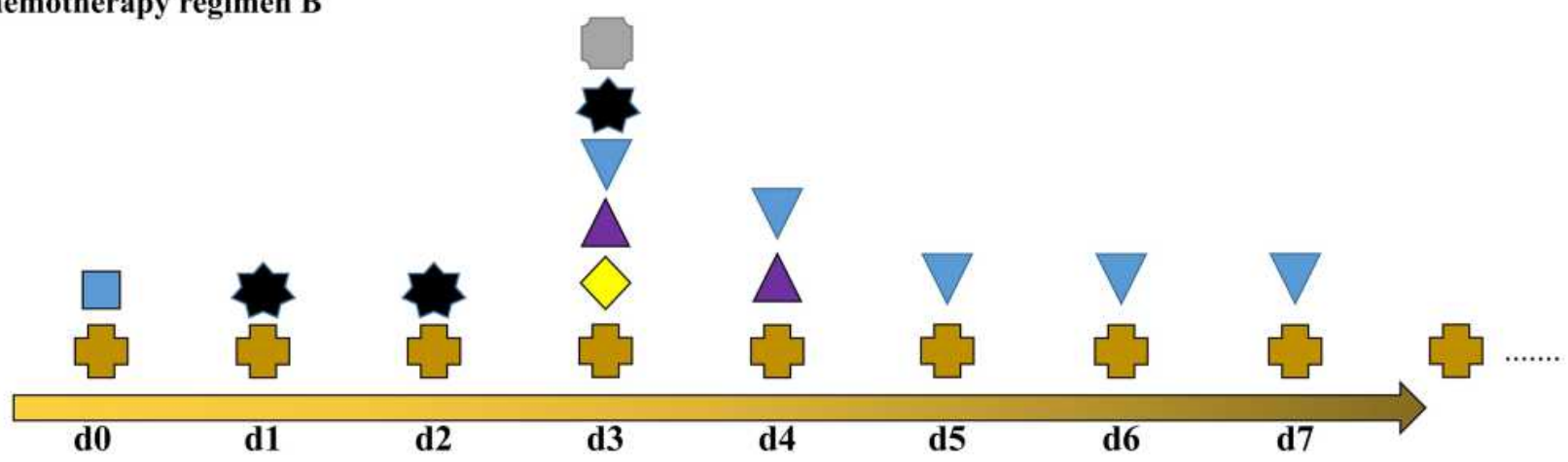

Figure 3 Schematic representation of chemotherapy regimens. Chemotherapy regimen A was applied to our patient in the first cycle and chemotherapy regimen B was adapted in the second to sixth cycle.

our patient suffered from diabetes which is a proven risk factor for high-dose methotrexate-associated AKI in lymphoma patients. ${ }^{11}$ Fotemustine, another alkylating agent, was also proved to be effective in glioma patients with MGMT promoter methylation compared to unmethylated MGMT. $^{12}$ Meanwhile, fotemustine, teniposide and dexamethasone (FTD) regimen has been applied in central nervous system lymphoma and been verified with no difference in 3 years PFS and OS when compared to HD-MA regimen (high-dose methotrexate plus cytarabine) in treating newly diagnosed primary central nervous system lymphoma. ${ }^{13}$ Our patient strongly believed that the massive amount of fluid infusion related to HD-MTX can interfere with her quality of life and refused to continue the regimen we designed in first cycle. In order to find a more suitable regimen, we referred previous clinical trials and her genetic features. Finally, we designed a new combination called chemotherapy regimen B (Figure 3, chemotherapy regimen $\mathrm{B})$. In this regimen, we replaced MTX and temozolomide with fotemustine $\left(140 \mathrm{mg} / \mathrm{m}^{2}, \mathrm{~d} 3\right)$ and teniposide $(65 \mathrm{mg} /$ $\mathrm{m}^{2}$ once a day, $\left.\mathrm{d} 1-3\right)$ in the second cycle of chemotherapy as the proved effect by clinical trials ${ }^{13}$ and we also added ibrutinib (420 mg once per day) to our patient's daily treatment in view of the $C D 79 B$ and $M y D 88$ L265P mutations in the tumors. ${ }^{14,15}$ In the meantime, previous research has confirmed methotrexate is antagonistic to ibrutinib. ${ }^{14}$ The ibrutinib-containing clinical trial (NCT03964090) for aggressive B-cell Lymphomas with secondary involvement of the central nervous system was also omit methotrexate because of the antagonistic effect. In summary, we changed our protocol to ibrutinib (420 mg once per day); rituximab (375 mg/m², d0); vincristine (2 mg, d3); cyclophosphamide $\left(0.75 \mathrm{~g} / \mathrm{m}^{2}\right.$ once per day, $\left.\mathrm{d} 3-4\right)$; prednisone $(60 \mathrm{mg}, \mathrm{d} 3-7)$; fotemustine $\left(140 \mathrm{mg} / \mathrm{m}^{2}, \mathrm{~d} 3\right)$ and teniposide $\left(65 \mathrm{mg} / \mathrm{m}^{2}\right.$ once a day, d1-3) (Figure 3, chemotherapy regimen B). An MRI scan was obtained before the third cycle of our new immunochemotherapy. The masses in her head were significantly reduced, which was judged as a PR (partial response) according to the Response Evaluation Criteria in Lymphoma (RECIL) 2017 standards $^{16}$ (Figure 1D-F), and she recovered from an unbearable headache. During the treatment period, our patient suffered from only Grade 1 fatigue and Grade 3 hematologic toxicity according to the Common Terminology Criteria for Adverse Events version 4. 
Unfortunately, after six cycles of treatment, our patient experienced severe myelosuppression and had an extremely low white cell count $\left(300 / \mathrm{mm}^{3}\right)$. Afterwards, she suffered from fulminant respiratory failure accompanied with low-grade fever and dry cough. Piperacillintazobactam treatment had no therapeutic efficacy, and she was arranged to be monitored and cared for in the intensive care unit (ICU). Her CT lung screening revealed pulmonary consolidation in the inferior lobes surrounded by a wide range of ground-glass opacity effusions on both sides (Figure 4). The beta-D-glucan assay test was positive and the sputum sample was negative for hyphae and spores. With the help of conventional mechanical ventilation, several broad-spectrum antibiotics, and antifungal therapy with voriconazole and trimethoprimsulfamethoxazole (TMP-SMX) were also added. Adjunctive dexamethasone $(5 \mathrm{mg} / \mathrm{d})$ was used to reduce the serious inflammatory conditions. We sent the patient's blood sample for the detection of potential pathogens by next-generation sequencing (NGS) technology as previous reported with her parents' permission. ${ }^{17}$ The results confirmed a PCP infection, and we increased the dose of TMP-SMX. A re-examination with chest CT after 10 days of treatment showed that the majority of the previous lesions had resolved, and her dyspnea improved without ventilatory support. Our patient recovered from PCP, and we rechecked her lymphoma status by PET/CT. There was no abnormal FDG uptake in the region that previously had high metabolic activity (Figure 5). Meanwhile, the lesions in the brain displayed the same metabolic signal as normal brain tissues. Bone marrow biopsy also confirmed complete remission after chemotherapy. We recommended that our patient receive another two cycles of rituximab injections. After finishing treatment, MRI was conducted again to evaluate the status in her head, and images revealed that the corpus callosum and ventricle lesions were much
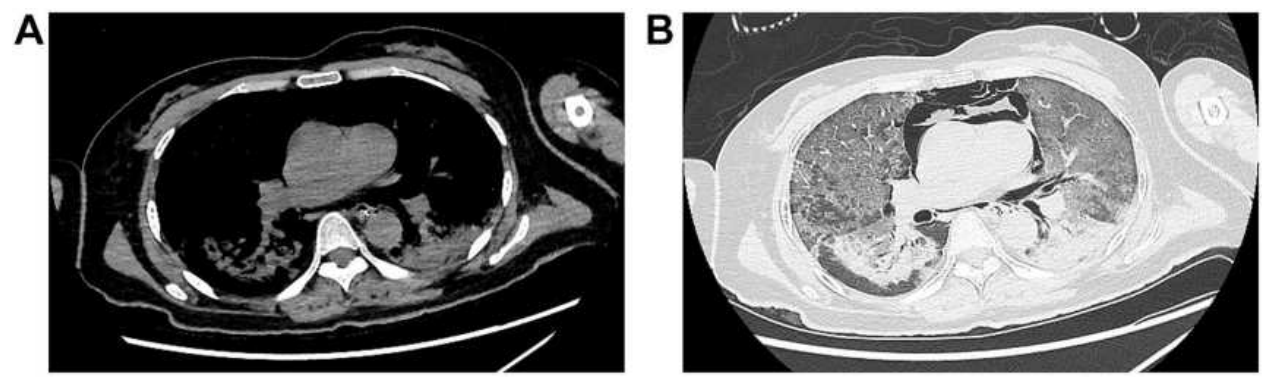

Figure 4 CT images after respiratory failure happened. Mediastinal emphysema was occurred after receiving invasive mechanical ventilation. Mediastinal window (A) and lung window (B).

A
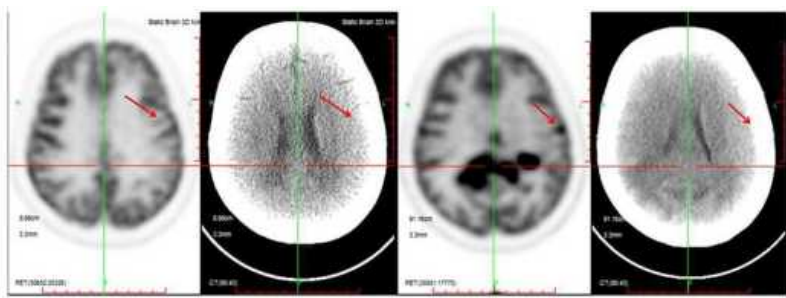

C
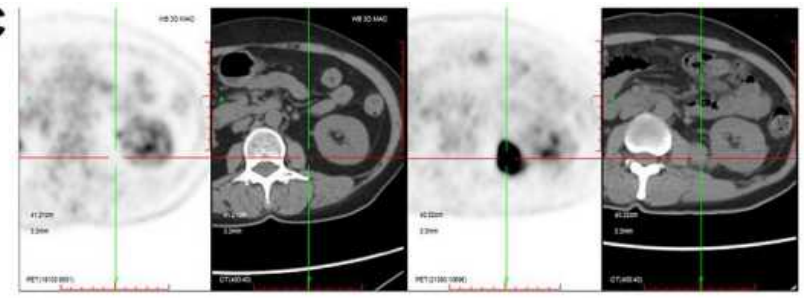

B
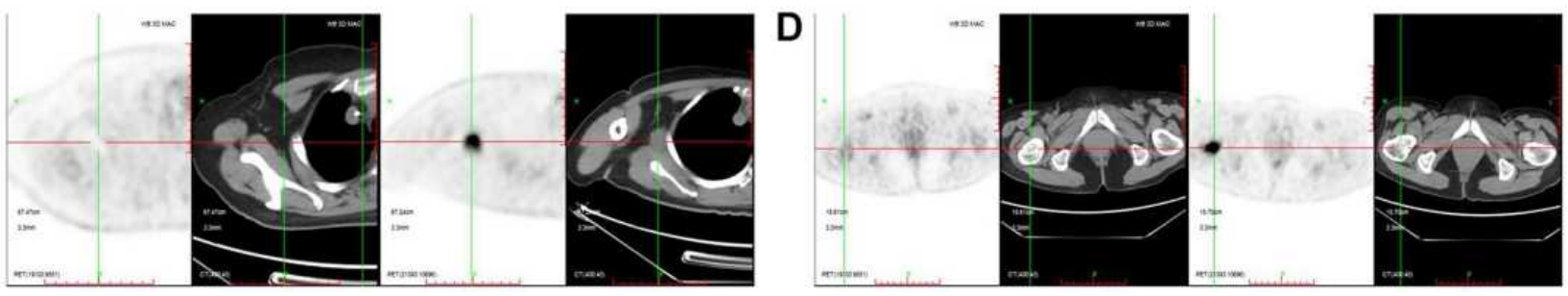

Figure $\mathbf{5}$ The changes of FDG uptake before and after treatment. FDG uptake signals disappeared in brain (A), axillary lymph nodes (B), lymph nodes around the kidney area (C) and right femoral neck (D) after our interventions. The red arrows showed a solitary lesion outside the ventricle, which was also disappeared after our treatment. 


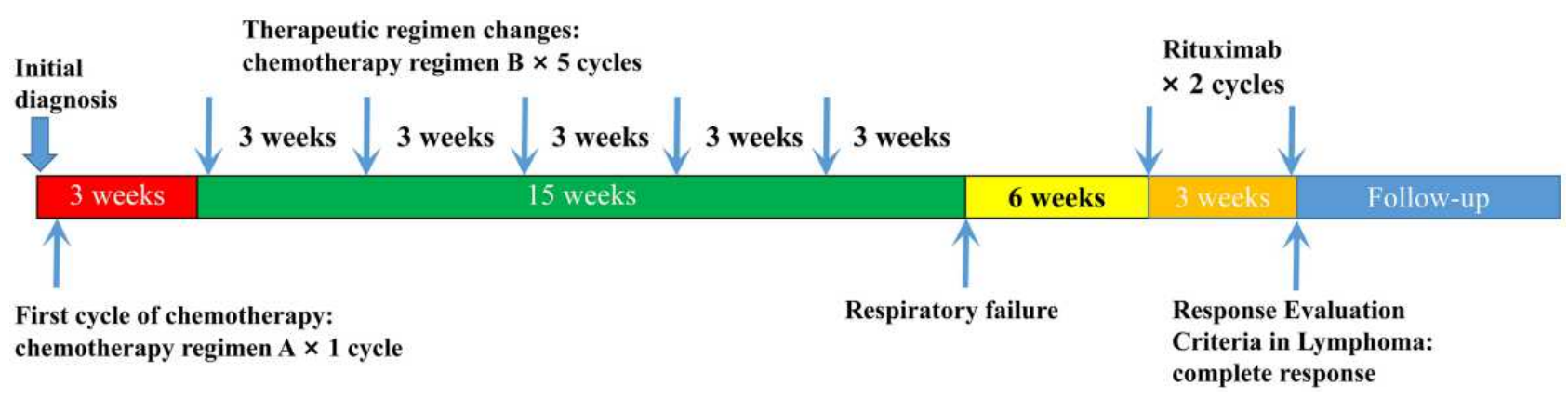

Figure 6 A Chart of the patient's medical procedures.

smaller than they were initially, and our patient was judged as complete response (Figure $1 \mathrm{G}-\mathrm{I}$ ) and our patient checked the MRI again after 30 months follow-up (Figure 1J-L). She refused further whole-brain radiation therapy (WBRT) or autologous stem cell transplantation (ASCT) for consolidation and continues to be followed. All the treatment procedures were shown in Figure 6.

\section{Discussion}

SCNSL is defined as lymphoma involvement both within and outside of the CNS at the time of diagnosis or as the CNS relapse of a systemic lymphoma ${ }^{18}$ and is a rare and fatal disease that often occurs in non-Hodgkin lymphoma. ${ }^{19}$ In our study, we described an SCNSL patient with a definitive diagnosis and individualized therapy. This case may help us establish a better, standard way to cure this kind of disease by combining clinical and genetic features.

Most pathological types of SCNSL can be categorized as DLBCL. To date, however, there has been little discussion about the treatment of SCNSL. In our case, high-dose MTX-based regimens were effective therapeutics for PCNSL, but they were not an excellent cure for disease outside of the $\mathrm{CNS}^{20}$ In turn, $\mathrm{R}-\mathrm{CHOP}$ regimens are standard treatments for aggressive B cell lymphoma, and none of these drugs can easily penetrate the blood-brain barrier. Until now, few studies have investigated the proper treatment method. A multicenter study directed by Ferreri, ${ }^{21}$ which is the largest published Phase II trial before we treated our patient, proposed a novel treatment with induction phase, intensification phase and consolidation phase. In short, R-CHOP was applied for one or two cycles to control extra-CNS disease at first. High-dose methotrexate and cytarabine combined with rituximab and intrathecal liposomal cytarabine were adapted for controlling lesions in central nervous system as induction phase. Intensification phase was only proceeded when patients achieved complete response (CR) or partial response $(\mathrm{PR})$ and the phase was consisted of R-HDS (rituximab, high doses of cyclophosphamide, cytarabine, and etoposide) regimen. Autologous stem-cell transplantation (ASCT) was done as consolidation. The results showed that the 2-year event-free survival (EFS) and 5 -year EFS were $50 \%$ and $40 \%$, respectively. Moreover, the 5-year overall survival (OS) of all included patients was $41 \%$; in particular, the patients who underwent HDASCT achieved a better 5-year OS (68\%). However, in a total of 38 patients, 10 patients still experienced treatment failure, the inclusion of three different types of B cell lymphoma in clinical trials created a selection bias, and the subtype of DLBCL was also unknown. Another prospective trial called MARIETTA study was published recently and was supported to be the largest trial for secondary CNS lymphoma until now. ${ }^{6,22}$ Treatment consisted of a sequential combination of two standardized chemotherapy regimens, MATRix (methotrexate, cytarabine, thiotepa, and rituximab) and RICE (rituximab, etoposide, ifosfamide, and carboplatin), followed by carmustinethiotepa and autologous HSCT as a consolidation therapy. Seventy-nine patients were enrolled, and 2-year progression-free survival was $71 \%$ in patients with CNS disease at initial lymphoma diagnosis. Although the result was encouraging, identification of the most effective treatment options in secondary CNS lymphoma was still unknown and more prospective clinical trials are needed to address the most effective and individualized treatment options.

MGMT promoter methylation was detected in our patient's tumor sample, and it has been frequently observed in high-grade glioma. ${ }^{23}$ MGMT promoter methylation is a good index for prognosis and indicates that 
tumors are more susceptible to alkylating agent-based therapies. $^{24}$ In DLBCL, MGMT promoter methylation indicates a favorable prognosis and was found in $75.9 \%$ of patient samples from the Middle East. ${ }^{25}$ Temozolomide as an alkylating agent is a widely used alkylating drug with rapid and nearly complete oral absorption ${ }^{26}$ that is recommended for the treatment of PCNSL. ${ }^{27}$ Because of its impressive curative effect, we added temozolomide in the first cycle of treatment with patient permission. However, our patient's complaints and several risk factors noticed above forced us to change to a more suitable regimen for her.

As we known, BCR is a very important transmembrane receptor that regulates $\mathrm{B}$ cell proliferation, differentiation and migration. ${ }^{28}$ An in vitro study conducted by Louis' group introduced a new pathway called chronic active BCR signaling that is distinct from tonic BCR signaling. ${ }^{29}$ Bruton's tyrosine kinase (BTK) is an essential molecule involved in BCR signaling and in vitro experiment confirmed that the BCR signaling pathway plays an essential role in ABC-type B cell malignancies.

Ibrutinib, a first-in-class inhibitor of BTK, was first available for mantle cell lymphoma and chronic lymphocytic leukemia (CLL). ${ }^{30,31}$ Because of the encouraging results in vitro, 80 patients with relapsed and refractory DLBCL were included in a study of oral ibrutinib. ${ }^{15}$ Interestingly, the response rate in the activated B-cell type was much higher than that in the germinal center B-cell type $(37 \%$ vs $5 \%, \mathrm{P}=0.0106)$. In a preclinical study, the distribution of ibrutinib was tested in mouse models. ${ }^{32}$ The results revealed that the ibrutinib concentration in the brain was strongly associated with the concentration in plasma. Furthermore, an effective concentration above the IC50 of ibrutinib was observed, which confirmed the therapeutic effect in intracranial lesions. Clinical research was also conducted by Louis and Wilson's group in which 18 patients were chosen, most of whom suffered from relapsed/refractory ABCtype PCNSL. ${ }^{14}$ Clinical trials confirmed that ibrutinib and its primary metabolite, PCI-45227, maintained a therapeutic concentration in CSF. The overall response rate (ORR) among patients was 94\% after treatment with ibrutinib combined with chemotherapy with temozolomide, etoposide, liposomal doxorubicin, dexamethasone and rituximab, which was defined as the DA-TEDDi-R regimen. Moreover, a previous study also confirmed that lymphomas with both MYD88 L265P and CD79B mutations were much more sensitive to ibrutinib treatment. ${ }^{15}$
Meanwhile, the combination of ibrutinib and MTX may decrease the efficacy of both drugs. ${ }^{14}$ We decided to add ibrutinib to the treatment schema and to replace MTX. A Phase 2 clinical trial also confirmed that the fotemustine, teniposide and dexamethasone combination achieved an ORR, 2-year progression-free survival (PFS) and 3-year OS that were similar to those of high-dose MTX plus cytarabine regimens in PCNSL patients. ${ }^{13}$ In the meantime, fotemustine is also an alkylating agent ${ }^{12}$ that may benefit our patient and we cannot find any regimens without MTX and containing temozolomide which have the same therapeutic effect in published data. In order to maintain both therapeutic effects after omitting MTX and the therapeutic regimens should include ibrutinib and alkylating agent according to genetic features of lymphoma, we changed our chemotherapy protocol as follows: ibrutinib (420 mg once per day); rituximab (375 mg/m², d0); vincristine $(2 \mathrm{mg}, \mathrm{d} 3)$; cyclophosphamide $\left(0.75 \mathrm{~g} / \mathrm{m}^{2}\right.$ once per day, d3-4); prednisone (60 $\mathrm{mg}, \mathrm{d} 3-7)$; fotemustine $\left(140 \mathrm{mg} / \mathrm{m}^{2}, \mathrm{~d} 3\right)$ and teniposide $\left(65 \mathrm{mg} / \mathrm{m}^{2}\right.$ once a day, d1-3) since the second cycle of chemotherapy (Figure 3, chemotherapy regimen B). Fortunately, our patient experienced remission after another five cycles of treatment (Figure 6).

Nevertheless, our patient suffered from a severe pulmonary infection after six cycles of therapy. Clinical laboratory examinations revealed that the presumptive diagnosis of the pathogen was Pneumocystis jirovecii. Several factors are known to be partially responsible for infections with Pneumocystis jirovecii. In a previous study, a missense mutation in the BTK gene caused a congenital disease called X-linked agammaglobulinemia (XLA), and patients with this disease were susceptible to Pneumocystis because of the Toll-like signaling deficiency that resembles the blocking effect of ibrutinib. ${ }^{33}$ This hypothesis was also proven by Ahn's group. ${ }^{34}$ Ninety-six patients suffering from CLL were treated with ibrutinib in a clinical trial, and five patients experienced the onset of PCP. The estimated incidence of PCP was 2.05 cases per 100 patientyears in previously untreated patients with CLL on singleagent ibrutinib. The prevalence of PCP was also proven by Ryan's group. $^{35}$ They pointed out that prevalence of PCP in BTKi-monotherapy patients not on prophylaxis was $2.4 \%$ (2 of 85 ). Additionally, our patient's immune system was impaired by high-intensity chemotherapy and was weakened by glucocorticoids. All these elements contributed to severe adverse reactions. However, it is controversial whether prophylaxis septrin should be given. 
Memorial Sloan Kettering Cancer Center guidelines recommend PCP prophylaxis for patients with lymphoid cancer who are receiving chemotherapy with a purine analogue or alemtuzumab or are receiving an average daily-dose equivalent to $\geq 20 \mathrm{mg}$ of prednisone for $\geq 4$ weeks. ${ }^{36}$ But there are no guidelines for ibrutinib-related treatment regimens. Previous study suggests that the prevalence of PCP is relatively low and routine prophylaxis may not be indicated, ${ }^{35}$ but the authors also showed that PCP prophylaxis was effective, as there were no cases of PCP in patients on prophylaxis. Additionally, by reviewing medical records of patients with lymphoid cancer at the Ohio State University, the most common type of infections during ibrutinib treatment was invasive fungal (61\%) other than PCP. ${ }^{37}$ Medical records at Memorial Sloan Kettering Cancer Center also confirmed that the much higher incidence of invasive fungal infections in patients with PCNSL compared to PCP. ${ }^{36}$ In our case, prophylaxis septrin may not be conventional in ibrutinib monotherapy. However, both cytotoxic chemotherapy and corticosteroids other than ibrutinib may also contribute our patient's PCP infections. Fortunately, after diagnosis, TMP-SMX was rapidly administered, and our patient recovered from respiratory failure.

\section{Conclusion}

We diagnosed a rare case of an SCNSL. With the help of whole-genome sequencing, ibrutinib and chemotherapeutics were included in our patient's treatment schedule. However, PCP occurred and caused respiratory failure in our patient. Although we diagnosed PCP in time and the patient recovered, we still note the possible danger of PCP, and TMP-SMX can be used as drug prophylaxis in chemotherapy regimens that include high-dose of cytotoxic chemotherapy and ibrutinib.

\section{Ethics Statement}

The written informed consent was obtained from the patient and the patient agreed to publish her details of her case and any accompanying images. This study was approved by the ethics committee of Xiangya Hospital, Central South University (Changsha, China) to publish the case details.

\section{Funding}

This work was supported in part by the National Natural Science Foundation of China (Grant No. 81900201) and
Youth Foundation of Xiangya Hospital (No. 2017Q09) to H.L.

\section{Disclosure}

The authors declare no conflicts of interest.

\section{References}

1. Bernstein SH, Unger JM, Leblanc M, Friedberg J, Miller TP, Fisher RI. Natural history of CNS relapse in patients with aggressive non-Hodgkin's lymphoma: a 20-year follow-up analysis of SWOG 8516 - the Southwest Oncology Group. J Clin Oncol. 2009;27 (1):114-119. doi:10.1200/JCO.2008.16.8021

2. Hollender A, Kvaloy S, Lote K, Nome O, Holte H. Prognostic factors in 140 adult patients with non-Hodgkin's lymphoma with systemic central nervous system (CNS) involvement. A single centre analysis. Eur J Cancer. 2000;36(14):1762-1768. doi:10.1016/S0959-8049(00) 00171-4

3. Hollender A, Kvaloy S, Nome O, Skovlund E, Lote K, Holte H. Central nervous system involvement following diagnosis of nonHodgkin's lymphoma: a risk model. Ann Oncol. 2002;13 (7):1099-1107. doi:10.1093/annonc/mdf175

4. Boehme V, Zeynalova S, Kloess M, et al. Incidence and risk factors of central nervous system recurrence in aggressive lymphoma-a survey of 1693 patients treated in protocols of the German highgrade non-Hodgkin's Lymphoma Study Group (DSHNHL). Ann Oncol. 2007;18(1):149-157. doi:10.1093/annonc/mdl327

5. Schmitz N, Zeynalova S, Nickelsen M, et al. CNS international prognostic index: a risk model for CNS relapse in patients with diffuse large B-cell lymphoma treated with R-CHOP. J Clin Oncol. 2016;34(26):3150-3156. doi:10.1200/JCO.2015.65.6520

6. Ferreri AJM, Doorduijn JK, Re A, et al. MATRix-RICE therapy and autologous haematopoietic stem-cell transplantation in diffuse large B-cell lymphoma with secondary CNS involvement (MARIETTA): an international, single-arm, phase 2 trial. Lancet Haematol. 2021;8 (2):e110-e121. doi:10.1016/S2352-3026(20)30366-5

7. Glass J, Won M, Schultz CJ, et al. Phase I and II study of induction chemotherapy with methotrexate, rituximab, and temozolomide, followed by whole-brain radiotherapy and postirradiation temozolomide for primary CNS lymphoma: NRG Oncology RTOG 0227. J Clin Oncol. 2016;34(14):1620-1625. doi:10.1200/JCO.2015.64.8634

8. Coiffier B, Lepage E, Briere J, et al. CHOP chemotherapy plus rituximab compared with $\mathrm{CHOP}$ alone in elderly patients with diffuse large-B-cell lymphoma. $N$ Engl $J$ Med. 2002;346(4):235-242. doi:10.1056/NEJMoa011795

9. DeAngelis LM, Seiferheld W, Schold SC, Fisher B, Schultz CJ. Combination chemotherapy and radiotherapy for primary central nervous system lymphoma: Radiation Therapy Oncology Group Study 93-10. J Clin Oncol. 2002;20(24):4643-4648. doi:10.1200/ JCO.2002.11.013

10. Ferreri AJ, Tarella C, Ciceri F. Reply to A. Korfel et al. J Clin Oncol. 2016;34(15):1830-1831. doi:10.1200/JCO.2016.66.4722

11. Wang Y, Wei L, Guan Y, Wang Q, Xie Q, Hao C. Diabetes is a risk factor for high-dose methotrexate-associated AKI in lymphoma patients. Ren Fail. 2020;42(1):1111-1117. doi:10.1080/0886022X.2020.1838926

12. Fabi A, Metro G, Russillo M, et al. Treatment of recurrent malignant gliomas with fotemustine monotherapy: impact of dose and correlation with MGMT promoter methylation. BMC Cancer. 2009;9:101. doi:10.1186/1471-2407-9-101

13. Wu J, Duan L, Zhang L, et al. Fotemustine, teniposide and dexamethasone versus high-dose methotrexate plus cytarabine in newly diagnosed primary CNS lymphoma: a randomised phase 2 trial. J Neurooncol. 2018;140(2):427-434. doi:10.1007/s11060-018-2970-x 
14. Lionakis MS, Dunleavy K, Roschewski M, et al. Inhibition of B cell receptor signaling by ibrutinib in primary CNS Lymphoma. Cancer Cell. 2017;31(6):833-843. doi:10.1016/j.ccell.2017.04.012

15. Wilson WH, Young RM, Schmitz R, et al. Targeting B cell receptor signaling with ibrutinib in diffuse large B cell lymphoma. Nat Med. 2015;21(8):922-926. doi:10.1038/nm.3884

16. Younes A, Hilden P, Coiffier B, et al. International Working Group consensus response evaluation criteria in lymphoma (RECIL 2017). Ann Oncol. 2017;28(7):1436-1447. doi:10.1093/annonc/mdx097

17. Ye M, Wei W, Yang Z, et al. Rapid diagnosis of Propionibacterium acnes infection in patient with hyperpyrexia after hematopoietic stem cell transplantation by next-generation sequencing: a case report. BMC Infect Dis. 2016;16:5. doi:10.1186/s12879-015-1306-0

18. Maciocia P, Badat M, Cheesman S, et al. Treatment of diffuse large B-cell lymphoma with secondary central nervous system involvement: encouraging efficacy using CNS-penetrating R-IDARAM chemotherapy. $\mathrm{Br}$ J Haematol. 2016;172(4):545-553. doi:10.1111/bjh.13867

19. Schmitz N, Wu HS. Advances in the treatment of secondary CNS lymphoma. J Clin Oncol. 2015;33(33):3851-3853. doi:10.1200/ JCO.2015.63.1143

20. Rubenstein JL, Gupta NK, Mannis GN, Lamarre AK, Treseler P. How I treat CNS lymphomas. Blood. 2013;122(14):2318-2330. doi:10.1182/blood-2013-06-453084

21. Ferreri AJ, Donadoni G, Cabras MG, et al. High doses of antimetabolites followed by high-dose sequential chemoimmunotherapy and autologous stem-cell transplantation in patients with systemic B-cell lymphoma and secondary CNS involvement: final results of a multicenter phase II trial. J Clin Oncol. 2015;33(33):3903-3910. doi:10.1200/JCO.2015.61.1236

22. Dietrich J. The best matrix for the brain: advances in secondary CNS lymphoma. Lancet Haematol. 2021;8(2):e96-e97. doi:10.1016/ S2352-3026(20)30431-2

23. Thon N, Kreth S, Kreth FW. Personalized treatment strategies in glioblastoma: MGMT promoter methylation status. Onco Targets Ther. 2013;6:1363-1372. doi:10.2147/OTT.S50208

24. Weller M, Stupp R, Reifenberger G, et al. MGMT promoter methylation in malignant gliomas: ready for personalized medicine? Nat Rev Neurol. 2010;6(1):39-51. doi:10.1038/nrneurol.2009.197

25. Al-Kuraya K, Narayanappa R, Siraj AK, et al. High frequency and strong prognostic relevance of O6-methylguanine DNA methyltransferase silencing in diffuse large B-cell lymphomas from the Middle East. Hum Pathol. 2006;37(6):742-748. doi:10.1016/j. humpath.2006.02.007
26. Dresemann G. Temozolomide in malignant glioma. Onco Targets Ther. 2010;3:139-146. doi:10.2147/OTT.S5480

27. Rubenstein JL, Hsi ED, Johnson JL, et al. Intensive chemotherapy and immunotherapy in patients with newly diagnosed primary CNS lymphoma: CALGB 50202 (Alliance 50202). J Clin Oncol. 2013;31 (25):3061-3068. doi:10.1200/JCO.2012.46.9957

28. Treanor B. B-cell receptor: from resting state to activate. Immunology. 2012;136(1):21-27. doi:10.1111/j.1365 2567.2012.03564.x

29. Davis RE, Ngo VN, Lenz G, et al. Chronic active B-cell-receptor signalling in diffuse large B-cell lymphoma. Nature. 2010;463 (7277):88-92. doi:10.1038/nature08638

30. Herrera AF, Jacobsen ED. Ibrutinib for the treatment of mantle cell lymphoma. Clin Cancer Res. 2014;20(21):5365-5371. doi:10.1158/ 1078-0432.CCR-14-0010

31. Byrd JC, Furman RR, Coutre SE, et al. Targeting BTK with ibrutinib in relapsed chronic lymphocytic leukemia. N Engl J Med. 2013;369 (1):32-42. doi:10.1056/NEJMoa1215637

32. Goldwirt L, Beccaria K, Ple A, Sauvageon H, Mourah S. Ibrutinib brain distribution: a preclinical study. Cancer Chemother Pharmacol. 2018;81(4):783-789. doi:10.1007/s00280-018-3546-3

33. Kanegane H, Nakano T, Shimono Y, Zhao M, Miyawaki T. Pneumocystis jiroveci pneumonia as an atypical presentation of X-linked agammaglobulinemia. Int J Hematol. 2009;89(5):716-717. doi:10.1007/s12185-009-0322-5

34. Ahn IE, Jerussi T, Farooqui M, Tian X, Wiestner A, Gea-Banacloche J. Atypical Pneumocystis jirovecii pneumonia in previously untreated patients with CLL on single-agent ibrutinib. Blood. 2016;128 (15):1940-1943. doi:10.1182/blood-2016-06-722991

35. Ryan CE, Cheng MP, Issa NC, Brown JR, Davids MS. Pneumocystis jirovecii pneumonia and institutional prophylaxis practices in CLL patients treated with BTK inhibitors. Blood Adv. 2020;4 (7):1458-1463. doi:10.1182/bloodadvances.2020001678

36. Varughese T, Taur Y, Cohen N, et al. Serious infections in patients receiving ibrutinib for treatment of lymphoid cancer. Clin Infect Dis. 2018;67(5):687-692. doi:10.1093/cid/ciy175

37. Rogers KA, Mousa L, Zhao Q, et al. Incidence of opportunistic infections during ibrutinib treatment for B-cell malignancies. Leukemia. 2019;33(10):2527-2530. doi:10.1038/s41375-019-0481-1
OncoTargets and Therapy

\section{Publish your work in this journal}

OncoTargets and Therapy is an international, peer-reviewed, open access journal focusing on the pathological basis of all cancers, potential targets for therapy and treatment protocols employed to improve the management of cancer patients. The journal also focuses on the impact of management programs and new therapeutic

Submit your manuscript here: https://www.dovepress.com/oncotargets-and-therapy-journal agents and protocols on patient perspectives such as quality of life, adherence and satisfaction. The manuscript management system is completely online and includes a very quick and fair peer-review system, which is all easy to use. Visit http://www.dovepress.com/ testimonials.php to read real quotes from published authors. 